\title{
SIMPLICITY OF ORE MONOID RINGS
}

\author{
PATRIK NYSTEDT \\ Department of Engineering Science, University West, SE-46186 Trollhättan, Sweden
}

JOHAN ÖINERT

Department of Mathematics and Natural Sciences, Blekinge Institute of Technology, SE-37179 Karlskrona, Sweden

\author{
JOHAN RICHTER \\ Academy of Education, Culture and Communication, Mälardalen University, Box 883, \\ SE-72123 Västerås, Sweden
}

\begin{abstract}
Given a non-associative unital $\operatorname{ring} R$, a monoid $G$ and a set $\pi$ of additive maps $R \rightarrow R$, we introduce the Ore monoid $\operatorname{ring} R[\pi ; G]$, and, in a special case, the differential monoid ring. We show that these structures generalize, in a natural way, not only the classical Ore extensions and differential polynomial rings, but also the constructions, introduced by Cojuhari, defined by so-called $D$-structures $\pi$. Moreover, for commutative monoids, we give necessary and sufficient conditions for differential monoid rings to be simple. We use this in a special case to obtain new and shorter proofs of classical simplicity results for differential polynomial rings in several variables previously obtained by Voskoglou and Malm by other means. We also give examples of new Ore-like structures defined by finite commutative monoids.
\end{abstract}

\section{INTRODUCTION}

1.1. Background. Suppose that $R$ is an associative unital ring with multiplicative identity 1. In [25] Ore introduced a version of skew polynomial rings $S=R[x ; \sigma, \delta]$, nowadays called Ore extensions, that have become a very important construction in ring theory. Recall that $S$ is defined to be the polynomial ring $R[x]$ as a left $R$-module, equipped with a new

E-mail addresses: patrik.nystedt@hv.se; johan.oinert@bth.se; johan.richter@mdh.se. 2010 Mathematics Subject Classification. 17D99, 17A36, 17A99.

Key words and phrases. non-associative Ore extension, iterated Ore extension, generalized monoid ring, Ore monoid ring, differential monoid ring, simple ring, outer derivation.

Corresponding author: Johan Öinert. 
associative multiplication induced by the relations $x r=\sigma(r) x+\delta(r)$, for $r \in R$, where $\sigma: R \rightarrow R$ is a ring endomorphism respecting 1 and $\delta: R \rightarrow R$ is a $\sigma$-derivation on $R$, that is $\delta$ is an additive map satisfying $\delta(r s)=\sigma(r) \delta(s)+\delta(r) s$ for all $r, s \in R$. In the special case when $\sigma=\mathrm{id}_{R}$, then $S$ is called a differential polynomial ring and $\delta$ is called a derivation. Ore extensions play an important role when investigating cyclic algebras, enveloping rings of solvable Lie algebras, and various types of graded rings such as group rings and crossed products (see e.g. [6, 15, 22, 26]). They are also a natural source of examples and counter-examples in ring theory (see e.g. [3, 5]). Furthermore, special cases of Ore extensions are used as tools in different analytical settings, such as differential-, pseudo-differential and fractional differential operator rings [11] and $q$-Heisenberg algebras [13].

Many different properties of Ore extensions, such as when they are integral domains, principal domains, prime or noetherian have been studied by numerous authors (see e.g. [10, 22, for surveys, or the articles [4, 17, 18, 19, 20]). For a moment, let us focus on the simplicity of a differential polynomial $\operatorname{ring} S=R\left[x ; \mathrm{id}_{R}, \delta\right]$. Recall that $\delta$ is called inner if there is $a \in R$ such that $\delta(r)=a r-r a$, for $r \in R$. In that case we write $\delta=\delta_{a}$. If $\delta$ is not inner, then $\delta$ is called outer. We let the characteristic of a ring $R$ be denoted by $\operatorname{char}(R)$. In an early article by Jacobson [14] it was shown that if $\delta$ is outer and $R$ is a division ring with $\operatorname{char}(R)=0$, then $S$ is simple. The case of positive characteristic is more complicated and $S$ may contain non-trivial ideals. In fact, Amitsur [1, 2] has shown that if $R$ is a simple ring with $\operatorname{char}(R)=p>0$, then every non-zero ideal of $D$ is generated by a polynomial, all of whose monomials have degrees which are multiples of $p$. It should be noticed that simplicity of $R$ is not a necessary condition for simplicity of $S=R\left[x ; \mathrm{id}_{R}, \delta\right]$. Consider e.g. the well known example of the first Weyl algebra where $R=K[y], K$ is a field with $\operatorname{char}(K)=0$ and $\delta$ is the usual derivative on $R$ (for more details, see e.g. [26, Example 1.6.32]). However, $\delta$-simplicity of $R$ is always a necessary condition for simplicity of $S$ (see [16, Lemma 4.1.3(i)]). Recall that an ideal $I$ of $R$ is called $\delta$-invariant if $\delta(I) \subseteq I$. The ring $R$ is called $\delta$-simple if $\{0\}$ and $R$ are the only $\delta$-invariant ideals of $R$. Jordan [16] (and Cozzens and Faith [10] in a special case) has generalized the results of Amitsur [2] to the case of $\delta$-simple $R$. In [24] Öinert, Richter and Silvestrov have shown that $S=R\left[x ; \operatorname{id}_{R}, \delta\right]$ is simple if and only if $R$ is $\delta$-simple and $Z(S)$ is a field. Simplicity results for differential polynomial rings in finitely many variables have been obtained by Voskoglou 28, and Malm 21. In 23] Nystedt, Öinert and Richter introduced a variant of Ore extensions $R[x ; \sigma, \delta]$ for non-associative (i.e. not necessarily associative) rings $R$ and weaker types of derivations. In loc. cit. simplicity results were obtained for these more general types of differential polynomial rings $R\left[x ; \mathrm{id}_{R}, \delta\right]$, thereby generalizing the results of [2, [16] and [24].

Many different generalizations of Ore extensions have been introduced. In [27] Smits considered skew polynomial rings $R[x]$ with a commutation rule defined by $x r=r_{1} x+$ $r_{2} x^{2}+\ldots+r_{n} x^{n}$, where $n \geq 2$ is a fixed integer and $r \in R$. In loc. cit. it was shown that the maps $\delta_{i}: r \mapsto r_{i}, i \in\{1, \ldots, n\}$, are additive. In the special case when $\delta_{i}=$ 0 , for $i \in\{2, \ldots, n\}$, one retrieves the skew polynomial ring $R[x ; \sigma, 0]$ where $\sigma=\delta_{1}$. 
Generalizations in several variables have also been considered. Let $\mathbb{N}$ denote the set of non-negative integers. If $n \in \mathbb{N}$ and $\delta_{0}, \ldots, \delta_{n}$ is a set of commuting derivations on $R$, then one can define the differential operator $\operatorname{ring} R\left[x_{0}, \ldots, x_{n} ; \delta_{0}, \ldots, \delta_{n}\right]$ as the ordinary polynomial ring $R\left[x_{0}, \ldots, x_{n}\right]$ subject to the relations $x_{i} x_{j}=x_{j} x_{i}$ and $x_{i} r=r x_{i}+\delta_{i}(r)$, for $i, j \in\{0, \ldots, n\}$ and $r \in R$ (see e.g. [28]). In [12] Hasse and Schmidt defined a higher derivation of rank $m \in \mathbb{N}$ as a sequence $d_{0}, d_{1}, \ldots, d_{m}$ of additive endomorphisms of $R$ satisfying the conditions $d_{n}(a b)=\sum_{i+j=n} d_{i}(a) d_{j}(b)$, for $n \in\{0, \ldots, m\}$, and $a, b \in R$. If the $d_{i}$ 's commute, then one can define a differential operator ring $R\left[x_{0}, \ldots, x_{m} ; d_{0}, \ldots d_{m}\right]$ as the ordinary polynomial ring $R\left[x_{0}, \ldots, x_{m}\right]$ subject to the relations $x_{i} x_{j}=x_{j} x_{i}$ and $x_{i} r=\sum_{k=0}^{i} d_{k}(r) x_{i-k}$, for $i, j \in\{0, \ldots, m\}$ and $r \in R$ (see e.g. [21]). In [7, 8, 9] Cojuhari and Gardner introduced skew versions $R[G ; \pi]$ of monoid rings in the following sense. Let $G$ denote a monoid with identity element $e$. For all $a, b \in G$, let $\pi_{b}^{a}: R \rightarrow R$ be a function. Following [8, Definition 1.1] we say that $\pi=\left\{\pi_{b}^{a}\right\}_{a, b \in G}$ is a D-structure on $R$ if the following axioms hold:

(D0) For all $a \in G$ and all $r \in R$, we have $\pi_{b}^{a}(r)=0$ for all but finitely many $b \in G$.

(D1) $\pi_{e}^{e}=\operatorname{id}_{R}$ and for all $a \in G \backslash\{e\}$, we have that $\pi_{a}^{e}=0$.

(D2) For all $a, b \in G, \pi_{b}^{a}(1)$ equals the Kronecker delta function $\delta_{a, b}$.

(D3) For all $a, b \in G$, the map $\pi_{b}^{a}$ is additive.

(D4) For all $a, b, c \in G$, we have $\pi_{c}^{a b}=\sum_{d e=c} \pi_{d}^{a} \circ \pi_{e}^{b}$.

(D5) For all $a, b \in G$ and all $r, s \in R$, we have $\pi_{b}^{a}(r s)=\sum_{c \in G} \pi_{c}^{a}(r) \pi_{b}^{c}(s)$.

The generalized monoid ring $R[G ; \pi]$ is defined to be the set of formal sums $\sum_{a \in G} r_{a} x^{a}$, where $r_{a} \in R$ is zero for all but finitely many $a \in G$. The addition on $R[G ; \pi]$ is defined pointwise and the multiplication on $R[G ; \pi]$ is defined by the biadditive extension of the relations

$$
\left(r x^{a}\right)\left(s x^{b}\right)=\sum_{c \in G} r \pi_{c}^{a}(s) x^{c b}
$$

for $r, s \in R$ and $a, b \in G$. It is not difficult to verify that $R[G ; \pi]$ is an associative unital ring (see [7, Section 4]). Notice that we recover the classical Ore extension $R[x ; \sigma, \delta]$ if we put $G=\mathbb{N}$ and let $\pi_{b}^{a}$ be the sum of all the $\left(\begin{array}{l}a \\ b\end{array}\right)$ possible compositions of $b$ copies of $\sigma$ and $a-b$ copies of $\delta$ in arbitrary order (see [25, Equation (11)] and [7, Example 2]). We also retrieve the differential operator rings defined by a higher derivation $d_{0}, d_{1}, d_{2}, \ldots, d_{m}$ which is iterative, that is such that $d_{a} \circ d_{b}=\left(\begin{array}{c}a+b \\ a\end{array}\right) d_{a+b}$ for all $a$ and $b$, if we put $\pi_{b}^{a}=(a ! / b !) d_{a-b}$, if $a \geq b$, and $\pi_{b}^{a}=0$, otherwise (see [8, Section 1]). Furthermore, crossed products, group rings and the construction of Smits [27] mentioned above can be obtained from this construction (see [7, Section 6]).

1.2. Outline. In this article, we consider Ore monoid rings $R[G ; \pi]$ in a context which is more general than the one studied by Cojuhari [7]. Indeed, we allow $R$ to be non-associative and do not necessarily require (D5) to hold. Consider the following axioms:

(D6) For all $a \in G$, the equality $\pi_{a}^{a}=\mathrm{id}_{R}$ holds.

(D7) For all $a, b \in G$, the map $\pi_{b}^{a}$ is left $R^{G}$-linear.

(D8) For all $a, b \in G$, the map $\pi_{b}^{a}$ is right $R^{G}$-linear. 
Here $R^{G}$ is defined to be the set of $r \in R$ such that for all $a, b \in G, \pi_{b}^{a}(r)=\delta_{a, b} r$. We say that $\pi$ is a (unital) $G$-derivation on $R$ if (D0)-(D4) (and (D6)) hold. In that case, $R[G ; \pi]$ is called an Ore monoid ring (differential monoid ring). The addition on $R[G ; \pi]$ is defined in the same way as for the generalized monoid ring, and the multiplication is defined by (11). We say that $\pi$ is a strong (unital) $G$-derivation on $R$ if also (D7) or (D8) holds. Moreover, we say that $\pi$ is a (unital) D-structure on $R$ if (D0)-(D5) (and (D6)) hold. In that case, $R[G ; \pi]$ is called a classical Ore monoid ring (classical differential monoid ring). It follows from the examples in [23] (for the case $G=\mathbb{N}$ ) that the following inclusions hold:

$$
\begin{gathered}
\text { \{classical Ore monoid rings }\} \subsetneq\{\text { Ore monoid rings }\} \\
\{\text { classical differential monoid rings }\} \subsetneq\{\text { differential monoid rings }\}
\end{gathered}
$$

The main objective of this article is to extend the simplicity results in [23] to Ore monoid rings. The secondary objective is to apply our extended results to particular cases of monoids to obtain new proofs of classical simplicity results for iterated Ore extensions as well as showing simplicity results for new Ore-like structures.

In Section 2, we show some results concerning commutativity and associativity in Ore monoid rings (see Propositions 28). At the end of the section, we describe certain elements in the center of Ore monoid rings, in the case when $G$ is commutative (see Corollary 10).

In Section [3, we show the main result of this article (see Theorem [18) which gives us necessary and sufficient conditions for simplicity of strong differential monoid rings $R[G ; \pi]$ under the hypothesis that $G$ is commutative and that there is a well-order $\preceq$ on $G$ with the property that for all $a, b \in G$ with $b \npreceq a$, the equality $\pi_{b}^{a}=0$ holds.

In Section 4, we apply the main result of the previous section to the monoid $\mathbb{N}^{(I)}$ of functions $f$ from a well-ordered set $I$ to $\mathbb{N}$, satisfying $f(i)=0$ for all but finitely many $i \in I$ (see Theorem 24). Using this result, we obtain generalizations of classical simplicity results by Voskoglou [28] and Malm [21] for differential polynomial rings in finitely many variables (see Theorem 33 and Theorem 35 ).

\section{THE CENTER}

Throughout this article, unless otherwise stated, $R$ denotes a unital non-associative ring and $S=R[G ; \pi]$ denotes an Ore monoid ring. Recall that the commutator $[\cdot, \cdot]: R \times R \rightarrow R$ and the associator $(\cdot, \cdot, \cdot): R \times R \times R \rightarrow R$ are defined by $[r, s]=r s-s r$ and $(r, s, t)=$ $(r s) t-r(s t)$ for all $r, s, t \in R$, respectively. The commuter of $R$, denoted by $C(R)$, is the subset of $R$ consisting of all elements $r \in R$ such that $[r, s]=0$, for all $s \in R$. The left, middle and right nucleus of $R$, denoted by $N_{l}(R), N_{m}(R)$ and $N_{r}(R)$, respectively, are defined by $N_{l}(R)=\{r \in R \mid(r, s, t)=0$, for $s, t \in R\}, N_{m}(R)=\{s \in R \mid(r, s, t)=$ 0 , for $r, t \in R\}$, and $N_{r}(R)=\{t \in R \mid(r, s, t)=0$, for $r, s \in R\}$. The nucleus of $R$, denoted by $N(R)$, is defined to be equal to $N_{l}(R) \cap N_{m}(R) \cap N_{r}(R)$. From the so-called associator identity $u(r, s, t)+(u, r, s) t+(u, r s, t)=(u r, s, t)+(u, r, s t)$, which holds for all $u, r, s, t \in R$, it follows that all of the subsets $N_{l}(R), N_{m}(R), N_{r}(R)$ and $N(R)$ are associative subrings of $R$. The center of $R$, denoted by $Z(R)$, is defined to be equal to the 
intersection $N(R) \cap C(R)$. It follows immediately that $Z(R)$ is an associative, unital and commutative subring of $R$.

Remark 1. If $a, b \in G$, then the additive map $\pi_{b}^{a}: R \rightarrow R$ may be extended to an additive map $\tilde{\pi}_{b}^{a}: S \rightarrow S$ by letting $\tilde{\pi}_{b}^{a}$ be the additive extension of the rule $\tilde{\pi}_{b}^{a}\left(r x^{c}\right)=\pi_{b}^{a}(r) x^{c}$, for $c \in G$ and $r \in R$. It is worth pointing out that, apart from additivity, $\tilde{\pi}_{b}^{a}$ need not enjoy any of the properties (e.g. (D4) or (D5)) of the original map $\pi_{b}^{a}$.

By defining $S^{G}$ analogously to how $R^{G}$ was defined, it is clear that $S^{G}=\sum_{a \in G} R^{G} x^{a}$.

Proposition 2. If $\pi$ is strong, then the following assertions hold:

(a) $R^{G}$ is a subring of $R$;

(b) $Z(R)^{G}$ is a commutative subring of $R$.

Proof. By assuming (D7) or (D8), the proof becomes straightforward.

Proposition 3. If $a \in G$, then $x^{a} \in N_{r}(S) \cap N_{m}(S)$.

Proof. Take $r, t \in R$ and $b, c \in G$. First we show that $x^{a} \in N_{r}(S):\left(r x^{b}, t x^{c}, x^{a}\right)=$ $\left(r x^{b} \cdot t x^{c}\right) x^{a}-r x^{b}\left(t x^{c} \cdot x^{a}\right)=\left(\sum_{d \in G} r \pi_{d}^{b}(t) x^{d c} \cdot x^{a}\right)-r x^{b} \cdot t x^{c a}=\sum_{d \in G} r \pi_{d}^{b}(t) x^{d c a}-$ $\sum_{d \in G} r \pi_{d}^{b}(t) x^{d c a}=0$. Next we show that $x^{a} \in N_{m}(S):\left(r x^{b}, x^{a}, t x^{c}\right)=\left(r x^{b} \cdot x^{a}\right) t x^{c}-$ $r x^{b}\left(x^{a} \cdot t x^{c}\right)=r x^{b a} \cdot t x^{c}-\sum_{d \in G} r x^{b} \cdot \pi_{d}^{a}(t) x^{d c}=\sum_{g \in G} r \pi_{g}^{b a}(t) x^{g c}-\sum_{d, f \in G} r\left(\pi_{f}^{b} \circ \pi_{d}^{a}\right)(t) x^{f d c}=$ $[(D 4)]=\sum_{g \in G} \sum_{f d=g} r\left(\pi_{f}^{b} \circ \pi_{d}^{a}\right)(t) x^{g c}-\sum_{d \in G} \sum_{f \in G} r\left(\pi_{f}^{b} \circ \pi_{d}^{a}\right)(t) x^{f d c}=0$.

Throughout the rest of this section, we shall assume that $G$ is commutative.

Proposition 4. If $s \in S^{G}$ and $a \in G$, then $\left[s, x^{a}\right]=0$.

Proof. Suppose that $s=\sum_{b \in G} r_{b} x^{b} \in S^{G}$. Then, for each $b \in G, r_{b} \in R^{G}$. Thus $\left[s, x^{a}\right]=$ $s x^{a}-x^{a} s=\sum_{b \in G} r_{b} x^{b a}-\sum_{b \in G} \sum_{c \in G} \pi_{c}^{a}\left(r_{b}\right) x^{c b}=\sum_{b \in G} r_{b} x^{b a}-\sum_{b \in G} r_{b} x^{a b}=0$.

Proposition 5. Suppose that $s \in S^{G}$. Then $[s, S] \subseteq[s, R] S$. In particular, if $[s, R]=\{0\}$, then $s \in C(S)$.

Proof. Suppose that $s=\sum_{a \in G} r_{a} x^{a} \in S^{G}$. Take $t \in R$ and $b \in G$. From Proposition 3 and Proposition 4, it follows that $\left[s, t x^{b}\right]=s\left(t x^{b}\right)-\left(t x^{b}\right) s=(s t) x^{b}-t\left(x^{b} s\right)=(s t) x^{b}-t\left(s x^{b}\right)=$ $(s t) x^{b}-(t s) x^{b}=[s, t] x^{b} \in[s, R] S$. The last part of the statement follows immediately.

Proposition 6. The following assertions hold:

(a) (Dr) holds if and only if for every $a \in G,\left(x^{a}, S^{G}, R\right)=\{0\}$;

(b) (D8) holds if and only if for every $a \in G,\left(x^{a}, R, S^{G}\right)=\{0\}$.

Proof. Take $a, b \in G, r \in R^{G}$ and $t \in R$.

(a) Suppose that (D7) holds. Then, using Proposition 3 and Proposition 4 we get that $\left(x^{a}, r x^{b}, t\right)=\left(x^{a} \cdot r x^{b}\right) t-x^{a}\left(r x^{b} \cdot t\right)=\left(\left(x^{a} r\right) x^{b}\right) t-x^{a}\left(r\left(x^{b} t\right)\right)=\left(r x^{a}\right)\left(x^{b} t\right)-x^{a}\left(r\left(x^{b} t\right)\right)=$ $\sum_{c \in G}\left(\left(r x^{a}\right)\left(\pi_{c}^{b}(t) x^{c}\right)-x^{a}\left(r \pi_{c}^{b}(t) x^{c}\right)\right)=\sum_{c \in G} \sum_{d \in G}\left(r \pi_{d}^{a}\left(\pi_{c}^{b}(t)\right) x^{d c}-\pi_{d}^{a}\left(r \pi_{c}^{b}(t)\right) x^{d c}\right)=0$. Now suppose that the equality $\left(x^{a}, S^{G}, R\right)=\{0\}$ holds for any $a \in G$. Then, from Proposition 3 and Proposition 4, we get that $0=\left(x^{a}, r, t\right)=\left(x^{a} r\right) t-x^{a}(r t)=\left(r x^{a}\right) t-$ $x^{a}(r t)=r\left(x^{a} t\right)-x^{a}(r t)=\sum_{c \in G}\left(r \pi_{c}^{a}(t) x^{c}-\pi_{c}^{a}(r t) x^{c}\right)$ from which (D7) follows. 
(b) Suppose that (D8) holds. We wish to show that $\left(x^{a}, t, r x^{b}\right)=0$. From Proposition 3 , it follows that it is enough to show this equality for $b=0$. Now, using Proposition 3 and Proposition 4. we get that $\left(x^{a}, t, r\right)=\left(x^{a} t\right) r-x^{a}(t r)=\sum_{c \in G}\left(\left(\pi_{c}^{a}(t) x^{c}\right) r-\pi_{c}^{a}(t r) x^{c}\right)=$ $\sum_{c \in G}\left(\pi_{c}^{a}(t) r-\pi_{c}^{a}(t r)\right) x^{c}=0$. On the other hand, if the equality $\left(x^{a}, R, S^{G}\right)=\{0\}$ holds, then, in particular, $0=\left(x^{a}, t, r\right)=\left(x^{a} t\right) r-x^{a}(t r)=\sum_{c \in G}\left(\left(\pi_{c}^{a}(t) x^{c}\right) r-\pi_{c}^{a}(t r) x^{c}\right)=$ $\sum_{c \in G}\left(\pi_{c}^{a}(t) r-\pi_{c}^{a}(t r)\right) x^{c}$, which shows that (D8) holds.

Proposition 7. Suppose that $s \in S$. Then $(s, S, S) \subseteq(s, R, R) S$. In particular, if $(s, R, R)=\{0\}$, then $s \in N_{l}(S)$.

Proof. Suppose that $s=\sum_{a \in G} r_{a} x^{a} \in S$. Take $r, t \in R$ and $b, c \in G$. We wish to show that $\left(s, r x^{b}, t x^{c}\right) \subseteq(s, R, R) S$. From Proposition [3, it follows that it is enough to prove this inclusion for $c=0$. Using Proposition 3 again, it follows that $\left(s, r x^{b}, t\right)=\left(s \cdot r x^{b}\right) t-$ $s\left(r x^{b} \cdot t\right)=\left((s r) x^{b}\right) t-s\left(r\left(x^{b} t\right)\right)=(s r)\left(x^{b} t\right)-\sum_{d \in G} s\left(r \pi_{d}^{b}(t) x^{d}\right)=\sum_{d \in G}(s r) \pi_{d}^{b}(t) x^{d}-$ $\sum_{d \in G}\left(s\left(r \pi_{d}^{b}(t)\right)\right) x^{d}=\sum_{d \in G}\left((s r) \pi_{d}^{b}(t)-s\left(r \pi_{d}^{b}(t)\right)\right) x^{d}=\sum_{d \in G}\left(s, r, \pi_{d}^{b}(t)\right) x^{d} \subseteq(s, R, R) S$. The last part of the statement follows immediately.

Proposition 8. Suppose that (D7) (or (D8)) holds and $s \in S^{G}$. Then $(S, s, S) \subseteq$ $(R, s, R) S($ or $(S, S, s) \subseteq(R, R, s) S)$. In particular, if $(R, s, R)=\{0\} \quad$ or $(R, R, s)=\{0\})$, then $s \in N_{m}(S)$ (or $s \in N_{r}(S)$ ).

Proof. Take $r, t \in R$ and $b, c \in G$. Suppose that (D7) holds. We wish to show that $\left(r x^{b}, s, t x^{c}\right) \subseteq(R, s, R) S$. From Proposition 3, it follows that it is enough to show this inclusion for $c=0$. Now, using Proposition 3, Proposition 4 and Proposition 6(a), we get that $\left(r x^{b}, s, t\right)=\left(r x^{b} \cdot s\right) t-r x^{b}(s \cdot t)=\left(r\left(x^{b} s\right)\right) t-r\left(x^{b}(s t)\right)=\left(r\left(s x^{b}\right)\right) t-r\left(\left(x^{b} s\right) t\right)=\left((r s) x^{b}\right) t-$ $r\left(\left(s x^{b}\right) t\right)=(r s)\left(x^{b} t\right)-r\left(s\left(x^{b} t\right)\right)=\sum_{d \in G}\left((r s) \pi_{d}^{b}(t) x^{d}-r\left(s \pi_{d}^{b}(t)\right) x^{d}\right)=\sum_{d \in G}\left((r s) \pi_{d}^{b}(t)-\right.$ $\left.r\left(s \pi_{d}^{b}(t)\right)\right) x^{d}=\sum_{d \in G}\left(r, s, \pi_{d}^{b}(t)\right) x^{d} \in(R, s, R) S$. The second part follows immediately. Now suppose that (D8) holds. We wish to show that $\left(r x^{b}, t x^{c}, s\right) \subseteq(R, R, s) S$. From Proposition 3 and Proposition 4, we get that $\left(r x^{b}, t x^{c}, s\right)=\left(r x^{b} \cdot t x^{c}\right) s-r x^{b}\left(t x^{c} \cdot s\right)=$ $\left(r x^{b} \cdot t\right)\left(x^{c} s\right)-r x^{b}\left(t\left(x^{c} s\right)\right)=\left(r x^{b} \cdot t\right)\left(s x^{c}\right)-r x^{b}\left(t\left(s x^{c}\right)\right)=\left(\left(r x^{b} \cdot t\right) s\right) x^{c}-r x^{b}\left((t s) x^{c}\right)=$ $\left(\left(r x^{b} \cdot t\right) s\right) x^{c}-\left(\left(r x^{b}\right)(t s)\right) x^{c}=\left(r x^{b}, t, s\right) x^{c}$. Thus, it is enough to prove the inclusion $\left(r x^{b}, t x^{c}, s\right) \subseteq(R, R, s) S$ for $c=0$. Now, using Proposition 3, Proposition 4 and Proposition 6(b), we get that

$$
\begin{aligned}
\left(r x^{b}, t, s\right) & =\left(r x^{b} \cdot t\right) s-r x^{b}(t \cdot s)=\left(r x^{b} \cdot t\right) s-r\left(x^{b}(t s)\right)=\left(r x^{b} \cdot t\right) s-r\left(\left(x^{b} t\right) s\right) \\
& =\sum_{d \in G}\left(\left(r \pi_{d}^{b}(t) x^{d}\right) s-r\left(\pi_{d}^{b}(t) x^{d} \cdot s\right)\right)=\sum_{d \in G}\left(\left(r \pi_{d}^{b}(t)\right)\left(x^{d} s\right)-r\left(\pi_{d}^{b}(t)\left(x^{d} s\right)\right)\right) \\
& =\sum_{d \in G}\left(\left(r \pi_{d}^{b}(t)\right)\left(s x^{d}\right)-r\left(\pi_{d}^{b}(t)\left(s x^{d}\right)\right)\right)=\sum_{d \in G}\left(\left(\left(r \pi_{d}^{b}(t)\right) s\right) x^{d}-\left(r\left(\pi_{d}^{b}(t) s\right)\right) x^{d}\right) \\
& =\sum_{d \in G}\left(\left(\left(r \pi_{d}^{b}(t)\right) s\right)-\left(r\left(\pi_{d}^{b}(t) s\right)\right)\right) x^{d}=\sum_{d \in G}\left(r, \pi_{d}^{b}(t), s\right) x^{d} \in(R, R, s) S .
\end{aligned}
$$

The second part follows immediately. 
Lemma 9. The following equalities hold:

$$
Z(S)=C(S) \cap N_{l}(S) \cap N_{m}(S)=C(S) \cap N_{l}(S) \cap N_{r}(S)=C(S) \cap N_{m}(S) \cap N_{r}(S)
$$

Proof. This holds for any non-associative ring $S$ (see e.g. [23, Proposition 2.1]).

Corollary 10. If $s \in S^{G}$ and $\pi$ is a strong $G$-derivation on $R$, then $s \in Z(S)$ if and only if $s$ commutes and associates with all elements of $R$.

Proof. The "only if" statement is immediate. The "if" statement follows from Proposition 7. Proposition 8 and Lemma 9.

\section{Simplicity}

In this section, we show the main result of this article (see Theorem 18). It gives us necessary and sufficient conditions for simplicity of a large class of differential monoid rings.

Definition 11. Let $I$ be an ideal of $R$. We say that $I$ is $G$-invariant if for all $a, b \in G$, the inclusion $\pi_{b}^{a}(I) \subseteq I$ holds. We say that $R$ is $G$-simple if $\{0\}$ and $R$ are the only $G$-invariant ideals of $R$. In Remark 1 we extended the maps $\pi_{b}^{a}: R \rightarrow R$, for $a, b \in G$, to additive maps $\tilde{\pi}_{b}^{a}: S \rightarrow S$. Using those extensions we may speak of $G$-simplicity of $S$.

Proposition 12. If $S$ is $G$-simple, then $R$ is G-simple.

Proof. Suppose that $I$ is a non-zero $G$-invariant ideal of $R$. Let $J$ denote the non-zero additive group $\sum_{g \in G} I x^{g}$. It is clear that $J$ is $G$-invariant. We claim that $J$ is an ideal of $S$. If we assume that the claim holds, then, by $G$-simplicity of $S$, we get that $J=S$, and, hence, that $I=R$. Now we show the claim. Take $r \in R, i \in I$ and $a, b \in G$. Since $I$ is a $G$-invariant ideal of $R$, we get that $\left(r x^{a}\right)\left(i x^{b}\right)=\sum_{c \in G} r \pi_{c}^{a}(i) x^{c b} \in \sum_{c \in G} R I x^{c b} \subseteq J$. On the other hand, we also get that $\left(i x^{b}\right)\left(r x^{a}\right)=\sum_{c \in G} i \pi_{c}^{b}(r) x^{c a} \in \sum_{c \in G} I R x^{c a} \subseteq J$.

Proposition 13. If $S$ is $G$-simple and for all $a, b \in G$, the map $\tilde{\pi}_{b}^{a}$ is right (left) $Z(S)^{G}$ linear, then $Z(S)^{G}$ is a field.

Proof. We show the "right" case. The "left" case is shown in an analogous fashion and is therefore left to the reader. It is not difficult to see that $Z(S)^{G}$ is a commutative ring. Take a non-zero $s \in Z(S)^{G}$. Let $I=S s$. Then $I$ is a non-zero ideal of $S$. Take $a, b \in G$. Then $\tilde{\pi}_{b}^{a}(I)=\tilde{\pi}_{b}^{a}(S s)=\tilde{\pi}_{b}^{a}(S) s \subseteq S s=I$. Therefore $I$ is $G$-invariant. By $G$-simplicity of $S$, we get that there is some $t \in S$ such that $s t=t s=1$. We know that $t \in Z(S)$. It remains to show that $t \in S^{G}$. Notice that $\tilde{\pi}_{a}^{a}(t)=\tilde{\pi}_{a}^{a}(t) s t=\tilde{\pi}_{a}^{a}(t s) t=\tilde{\pi}_{a}^{a}(1) t=1 t=t$, and if $a \neq b$, then $\tilde{\pi}_{b}^{a}(t)=\tilde{\pi}_{b}^{a}(t) s t=\tilde{\pi}_{b}^{a}(t s) t=\tilde{\pi}_{b}^{a}(1) t=0 t=0$.

Proposition 14. If $a, b \in G$ and $\pi_{b}^{a}$ is right $R^{G}$-linear, then $\tilde{\pi}_{b}^{a}$ is right $S^{G}$-linear.

Proof. If $a, b, d, g \in G, r \in R$ and $s \in R^{G}$, then $\tilde{\pi}_{b}^{a}\left(r x^{d} \cdot s x^{g}\right)=\tilde{\pi}_{b}^{a}\left(\sum_{c \in G} r \pi_{c}^{d}(s) x^{c g}\right)=$ $\tilde{\pi}_{b}^{a}\left(r s x^{d g}\right)=\pi_{b}^{a}(r s) x^{d g}=\pi_{b}^{a}(r) s x^{d g}=\sum_{c \in G} \pi_{b}^{a}(r) \pi_{c}^{d}(s) x^{c g}=\pi_{b}^{a}(r) x^{d} \cdot s x^{g}=\tilde{\pi}_{b}^{a}\left(r x^{d}\right) s x^{g}$.

Definition 15. We say that $\pi$ is commutative if $\pi_{b}^{a} \circ \pi_{d}^{c}=\pi_{d}^{c} \circ \pi_{b}^{a}$, for all $a, b, c, d \in G$. 


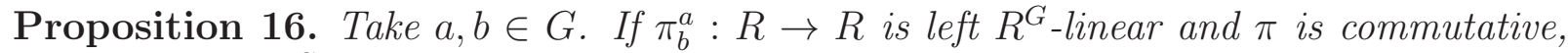
then $\tilde{\pi}_{b}^{a}$ is left $S^{G}$-linear.

Proof. If $d, e \in G, r \in R$ and $s \in R^{G}$, then $\tilde{\pi}_{b}^{a}\left(s x^{e} \cdot r x^{d}\right)=\tilde{\pi}_{b}^{a}\left(\sum_{c \in G} s \pi_{c}^{e}(r) x^{c d}\right)=$ $\sum_{c \in G} \pi_{b}^{a}\left(s \pi_{c}^{e}(r)\right) x^{c d}=\sum_{c \in G} s \pi_{b}^{a}\left(\pi_{c}^{e}(r)\right) x^{c d}=\sum_{c \in G} s \pi_{c}^{e}\left(\pi_{b}^{a}(r)\right) x^{c d}=s x^{e} \cdot \pi_{b}^{a}(r) x^{d}=s x^{e}$. $\tilde{\pi}_{b}^{a}\left(r x^{d}\right)$.

Definition 17. We say that $\pi$ is well-ordered if there is a well-order $\preceq$ on $G$ with the property that if $a, b \in G$ satisfy $a \prec b$, then $\pi_{b}^{a}=0$ (here, $a \prec b$ means that $a \preceq b$ and $a \neq b)$. In that case, we can define the degree map deg $: S \backslash\{0\} \rightarrow G$ in the following way. Take a non-zero element $s=\sum_{g \in G} r_{g} x^{g} \in S$. Since $\operatorname{supp}(s):=\left\{g \in G \mid r_{g} \neq 0\right\}$ is finite, $\operatorname{supp}(s)$ has a greatest element with respect to $\preceq$. That element will be denoted by $\operatorname{deg}(s)$.

Theorem 18. Suppose that $S=R[G ; \pi]$ is a differential monoid ring with $G$ commutative, and $\pi$ strong and well-ordered. If (D8) holds (or (DY) holds and $\pi$ is commutative), then $S$ is $G$-simple if and only if $R$ is $G$-simple and $Z(S)^{G}$ is a field.

Proof. The "only if" direction follows from Proposition 12, Proposition 13, Proposition 14 and Proposition 16. Now we show the "if" direction (using either assumption). Suppose that $R$ is $G$-simple and that $Z(S)^{G}$ is a field. Take a non-zero $G$-invariant ideal $I$ of $S$. Since $\preceq$ is a well-order we can define the least degree $m$ of non-zero elements of $I$. Define the non-empty subset $J$ of $R$ by saying that $r \in J$ if there are $r_{g} \in R$, for $g \in G$, with $g \prec m$, such that $r x^{m}+\sum_{g \prec m} r_{g} x^{g} \in I$. It is clear that $J$ is a non-zero left ideal of $R$. Using that $\pi$ is well-ordered and $S$ is a differential monoid ring, it follows that $J$ is also a right ideal of $R$. Since $I$ is $G$-invariant it follows that $J$ is $G$-invariant. From $G$-simplicity of $R$ we get that $J=R$. In particular, we get that there are $r_{g} \in R$, for $g \in G$ with $g \prec m$, such that $y:=x^{m}+\sum_{g \prec m} r_{g} x^{g} \in I$. Since $I$ is $G$-invariant, the minimality of $m$ yields that $y \in S^{G}$. Take $r \in R$. Put $z=r y-y r$. Then $\operatorname{deg}(z) \prec m$ which implies that $z=0$, since $z \in I$. Thus, $y$ commutes with all elements of $R$. Next we show that $y \in N(S)$. By Proposition 10, it is enough to show that $y$ associates with all elements of $R$. Take $r, s \in R$. It is easy to see that the degrees of all the elements $(y, r, s),(r, y, s)$ and $(r, s, y)$ are less than $m$. Hence, by minimality of $m$, we get that they are all zero. Therefore, $y$ is a non-zero element in the field $Z(S)^{G}$. This implies that $I=S$. Thus, $S$ is $G$-simple.

Example 19. In this example, $R$ denotes a unital non-associative algebra over a field $F$ such that $F \subseteq R$ and $1=1_{R} \in F$. Furthermore, $S=R[G ; \pi]$ denotes the corresponding differential monoid ring where $\pi$ is assumed to be a unital $G$-derivation.

Suppose that $G=\{0, g\}$ is the unique cyclic monoid with two elements not forming a group. Furthermore, suppose that $\pi_{0}^{g} \neq 0$. Then $g+g=g$. Notice that there is a natural well-order on $G$. From (D2), (D4) and (D6) it follows that $\pi_{0}^{0}=\pi_{g}^{g}=\mathrm{id}_{R}, \pi_{0}^{g}(1)=0$, $\pi_{0}^{g} \circ \pi_{0}^{g}=\pi_{0}^{g}$, and $\operatorname{char}(F)=2$. The last conclusion follows from the equality

$$
\mathrm{id}_{R}=\pi_{g}^{g}=\pi_{g}^{g+g}=\pi_{g}^{g} \circ \pi_{0}^{g}+\pi_{0}^{g} \circ \pi_{g}^{g}+\pi_{g}^{g} \circ \pi_{g}^{g}
$$

which simplifies to $\mathrm{id}_{R}=2 \pi_{0}^{g}+\mathrm{id}_{R}$.

Put $P=\pi_{0}^{g}$. We can write $R=\operatorname{ker}(P) \oplus V$ for some $F$-vector subspace $V$ of $R$ and notice that $R^{G}=\operatorname{ker}(P)=F$. Thus, it follows that $\pi_{0}^{g}$ is both right and left $R^{G}$-linear. 
Hence $\pi$ is strong. It is easy to check that $Z(S)^{G}=F$. By Theorem 18, $S$ is $G$-simple if and only if $R$ is $G$-simple. It now becomes easy to construct examples of simple nonassociative differential monoid rings. Indeed, let $Q: R \rightarrow F$ denote the projection. If for any non-zero $r \in R$, there is $r^{\prime} \in R$ such that $Q\left(r r^{\prime}\right) \neq 0$ or $Q\left(r^{\prime} r\right) \neq 0$, then $S$ is simple.

\section{THE MONOID $\mathbb{N}^{(I)}$}

Let $\mathbb{N}$ denote the set of non-negative integers. If $m, n \in \mathbb{N}$ satisfy $m \geq n$, then we let $\left(\begin{array}{l}m \\ n\end{array}\right)$ have its usual meaning. If $m, n \in \mathbb{N}$ satisfy $m<n$, then we put $\left(\begin{array}{c}m \\ n\end{array}\right)=0$. Throughout this section, let $I$ be a set which is well-ordered with respect to a relation $\ll$. For a function $f: I \rightarrow \mathbb{N}$ we let $\operatorname{supp}(f)$ denote the support of $f$, i.e. the set $\{i \in I \mid f(i) \neq 0\}$. Let $\mathbb{N}^{(I)}$ denote the set of functions $I \rightarrow \mathbb{N}$ with finite support. Take $f, g \in \mathbb{N}^{(I)}$. Define $f+g \in \mathbb{N}^{(I)}$ from the relations $(f+g)(i)=f(i)+g(i)$, for $i \in I$. With this operation $\mathbb{N}^{(I)}$ is a commutative monoid. Furthermore, put $\left(\begin{array}{l}f \\ g\end{array}\right)=\prod_{i \in I}\left(\begin{array}{c}f(i) \\ g(i)\end{array}\right)$. We define a partial order $\leq$ on $\mathbb{N}^{(I)}$ by saying that $f \leq g$ if, for all $i \in I$, the relation $f(i) \leq g(i)$ holds. Notice that if $f \leq g$, then the pointwise subtraction $g-f$ belongs to $\mathbb{N}^{(I)}$. Given $f \in \mathbb{N}^{(I)}$, with cardinality of $\operatorname{supp}(f)$ equal to $m \in \mathbb{N}$, we will often, for simplicity of notation, assume that $\operatorname{supp}(f)=\{1, \ldots, m\} \subseteq I$.

Definition 20. Suppose that for each $i \in I, \delta_{i}: R \rightarrow R$ is an additive map satisfying $\delta_{i}(1)=0$. Put $\Delta=\left\{\delta_{i}\right\}_{i \in I}$ and $R_{\Delta}=\bigcap_{i \in I} \operatorname{ker}\left(\delta_{i}\right)$. An ideal $J$ of $R$ is said to be $\Delta$-invariant if for each $i \in I, \delta_{i}(J) \subseteq J$. If $\{0\}$ and $R$ are the only $\Delta$-invariant ideals of $R$, then $R$ is said to be $\Delta$-simple. We say that $\Delta$ is commutative if for all $i, j \in I$, the relation $\delta_{i} \circ \delta_{j}=\delta_{j} \circ \delta_{i}$ holds. Furthermore, $\Delta$ is said to be a set of left (right) kernel derivations on $R$ if for each $i \in I, \delta_{i}$ is left (right) $R_{\Delta}$-linear. Take $f, g \in \mathbb{N}^{(I)}$ and $m \in \mathbb{N}$ such that the support of $f$ is contained in $\{1, \ldots, m\}$. Define $\delta^{f}: R \rightarrow R$ by $\delta^{f}(r)=\left(\delta_{1}^{f(1)} \circ \cdots \circ \delta_{m}^{f(m)}\right)(r)$, for $r \in R$. Furthermore we define $\pi_{g}^{f}: R \rightarrow R$ by $\pi_{g}^{f}(r)=\left(\begin{array}{l}f \\ g\end{array}\right) \delta^{f-g}(r)$, for $r \in R$. Notice that if $g \not \leq f$, then $f-g$ is not defined as an element of $\mathbb{N}^{(I)}$. However, since, in that case, $\left(\begin{array}{l}f \\ g\end{array}\right)=0$, the term $\pi_{g}^{f}(r)$ is supposed to be interpreted as 0 .

Proposition 21. With the above notation the following assertions hold:

(a) $\pi$ satisfies (D0), (D1), (D2) and (D6);

(b) $\pi$ satisfies (D4) $\Leftrightarrow \pi$ is commutative $\Leftrightarrow \Delta$ is commutative;

(c) Suppose that $\Delta$ is commutative. Then, $\pi$ satisfies (D5) $\Leftrightarrow$ each $\delta_{i}, i \in I$, is a derivation on $R$;

(d) $R^{\mathbb{N}^{(I)}}=R_{\Delta}$;

(e) $\pi$ satisfies (Dy) (or (D8)) $\Leftrightarrow \Delta$ is a set of left (or right) kernel derivations on $R$;

(f) Suppose that $\Delta$ is commutative. Then $R$ is $\Delta$-simple $\Leftrightarrow R$ is $\mathbb{N}^{(I)}$-simple.

Proof. (a) and (b) follow immediately from the definition of $\pi$.

(c) Suppose that (D5) holds. Take $i \in I$ and define $f_{i} \in \mathbb{N}^{(I)}$ by the relations $f_{i}(j)=$ $\delta_{i, j}$, for $j \in I$. For any $r, s \in R$ we get $\delta_{i}(r s)=\left(\begin{array}{c}f_{i} \\ 0\end{array}\right) \delta^{f_{i}}(r s)=\pi_{0}^{f_{i}}(r s)=\pi_{0}^{f_{i}}(r) \pi_{0}^{0}(s)+$ $\pi_{0}^{0}(r) \pi_{0}^{f_{i}}(s)=\delta_{i}(r) s+r \delta_{i}(s)$, which implies that $\delta_{i}$ is a derivation on $R$. 
Now suppose that for each $i \in I, \delta_{i}$ is a derivation on $R$. We first prove that, for any $i \in I, r, s \in R$ and $n \in \mathbb{N}$, the equation

$$
\delta_{i}^{n}(r s)=\sum_{k=0}^{n}\left(\begin{array}{l}
n \\
k
\end{array}\right) \delta^{n-k}(r) \delta^{k}(s)
$$

holds. Take $i \in I$ and $r, s \in R$. Equation (2) clearly holds if $n=0$ or $n=1$. We will prove the general case by induction. To this end, suppose that Equation (2) holds for $n$. For clarity, we will write $\delta$ instead of $\delta_{i}$. We now get

$$
\begin{aligned}
\delta^{n+1}(r s) & =\delta\left(\delta^{n}(r s)\right)=\delta\left(\sum_{k=0}^{n}\left(\begin{array}{l}
n \\
k
\end{array}\right) \delta^{n-k}(r) \delta^{k}(s)\right) \\
& =\left(\sum_{k=0}^{n}\left(\begin{array}{l}
n \\
k
\end{array}\right)\left(\delta^{n+1-k}(r) \delta^{k}(s)+\delta^{n-k}(r) \delta^{k+1}(s)\right)\right) \\
& =\sum_{k=0}^{n}\left(\begin{array}{l}
n \\
k
\end{array}\right) \delta^{n+1-k}(r) \delta^{k}(s)+\sum_{k=0}^{n}\left(\begin{array}{l}
n \\
k
\end{array}\right) \delta^{n-k}(r) \delta^{k+1}(s) \\
& =\sum_{k=0}^{n}\left(\begin{array}{l}
n \\
k
\end{array}\right) \delta^{n+1-k}(r) \delta^{k}(s)+\sum_{k=1}^{n+1}\left(\begin{array}{c}
n \\
k-1
\end{array}\right) \delta^{n+1-k}(r) \delta^{k}(s) \\
& \left.=\sum_{k=0}^{n+1}\left(\begin{array}{l}
n \\
k
\end{array}\right)+\left(\begin{array}{c}
n \\
k-1
\end{array}\right)\right) \delta^{n+1-k}(r) \delta^{k}(s)=\sum_{k=0}^{n+1}\left(\begin{array}{c}
n+1 \\
k
\end{array}\right) \delta^{n+1-k}(r) \delta^{k}(s)
\end{aligned}
$$

and by induction we conclude that Equation (2) holds for any $n \in \mathbb{N}$.

We will first show (D5) in a special case. Suppose that $f(i)=n, g(i)=m \leq n$ and $f(j)=g(j)=0$ if $i \neq j$. Then we get

$$
\begin{aligned}
\pi_{g}^{f}(r s) & =\left(\begin{array}{c}
n \\
m
\end{array}\right) \delta^{n-m}(r s)=\left(\begin{array}{c}
n \\
m
\end{array}\right) \sum_{k=0}^{n-m}\left(\begin{array}{c}
n-m \\
k
\end{array}\right) \delta^{n-m-k}(r) \delta^{k}(s) \\
& =\sum_{k=0}^{n-m}\left(\begin{array}{l}
n \\
m
\end{array}\right)\left(\begin{array}{c}
n-m \\
k
\end{array}\right) \delta^{n-m-k}(r) \delta^{k}(s)=\sum_{k=m}^{n}\left(\begin{array}{c}
n \\
m
\end{array}\right)\left(\begin{array}{c}
n-m \\
k-m
\end{array}\right) \delta^{n-k}(r) \delta^{k-m}(s) \\
& =\sum_{k=m}^{n}\left(\begin{array}{l}
n \\
m
\end{array}\right)\left(\begin{array}{c}
n-m \\
n-k
\end{array}\right) \delta^{n-k}(r) \delta^{k-m}(s)=\sum_{k=m}^{n}\left(\begin{array}{c}
n \\
n-k
\end{array}\right)\left(\begin{array}{c}
k \\
m
\end{array}\right) \delta^{n-k}(r) \delta^{k-m}(s) \\
& =\sum_{k=m}^{n}\left(\begin{array}{l}
n \\
k
\end{array}\right)\left(\begin{array}{c}
k \\
m
\end{array}\right) \delta^{n-k}(r) \delta^{k-m}(s)=\sum_{k=m}^{n}\left(\begin{array}{c}
n \\
k
\end{array}\right) \delta^{n-k}(r)\left(\begin{array}{c}
k \\
m
\end{array}\right) \delta^{k-m}(s)=\sum_{h \in \mathbb{N}(I)} \pi_{h}^{f}(r) \pi_{g}^{h}(s) .
\end{aligned}
$$

In the above calculation we have used an identity for binomial coefficients that will be generalized in Proposition 29.

We have proved that (D5) holds for $\pi_{g}^{f}$ if the support of $f$ only contains one element. The general case can be proved by induction on the size of the support. To this end, suppose that we have proved (D5) if the functions involved have support of size at most $n$. Let $f$ be 
a function with a support of size $n+1$ and let $g \leq f$. We can write $f=f^{\prime}+\bar{f}$ and $g=g^{\prime}+\bar{g}$, where the support of $f^{\prime}$ and $\bar{f}$ are disjoint, the support of $\bar{f}$ has size $n$, the support of $f^{\prime}$ has size 1 and $g^{\prime} \leq f^{\prime}$ and $\bar{g} \leq \bar{f}$. Then $\pi_{g}^{f}=\pi_{g^{\prime}}^{f^{\prime}} \circ \pi_{\bar{g}}^{\bar{f}}$. By the induction hypothesis we have $\pi_{g}^{f}(r s)=\pi_{g^{\prime}}^{f^{\prime}} \circ \pi_{\bar{g}}^{\bar{f}}(r s)=\pi_{g^{\prime}}^{f^{\prime}}\left(\sum_{\bar{h}} \pi_{\bar{h}}^{\bar{f}}(r) \pi_{\bar{g}}^{\bar{h}}(s)\right)=\sum_{\bar{h}} \sum_{h^{\prime}} \pi_{h^{\prime}}^{f^{\prime}}\left(\pi_{\bar{h}}^{\bar{f}}(r)\right) \pi_{g^{\prime}}^{h^{\prime}}\left(\pi_{\bar{g}}^{\bar{h}}(s)\right)=$ $\sum_{g \leq h^{\prime}+\bar{h} \leq f} \pi_{h^{\prime}}^{f^{\prime}}\left(\pi_{\bar{h}}^{\bar{f}}(r)\right) \pi_{g^{\prime}}^{h^{\prime}}\left(\pi_{\bar{g}}^{\bar{h}}(s)\right) \sum_{g \leq h \leq f} \pi_{h}^{f}(r) \pi_{g}^{h}(s)=\sum_{h} \pi_{h}^{f}(r) \pi_{g}^{h}(s)$.

(d) First we show the inclusion $R^{\mathbb{N}^{(I)}} \supseteq R_{\Delta}$. Take $r \in R_{\Delta}$. Then, for each $i \in I$, the equality $\delta_{i}(r)=0$ holds. Take $f, g \in \mathbb{N}^{(I)}$ such that $f \geq g$. Then, from the definition of $\pi_{g}^{f}$, it follows that $\pi_{g}^{f}(r)=0$. Thus, $r \in R^{\mathbb{N}^{(I)}}$. Now we show the converse inclusion $R^{\mathbb{N}^{(I)}} \subseteq R_{\Delta}$. Take $r \in R^{\mathbb{N}^{(I)}}$ and $i \in I$. Define $f_{i} \in \mathbb{N}^{(I)}$ by the relations $f_{i}(j)=1$, if $j=i$, and $f_{i}(j)=0$, otherwise. Since $r \in R^{\mathbb{N}^{(I)}}$, we get, in particular, that $0=\pi_{0}^{f_{i}}(r)=\left(\begin{array}{c}f_{i} \\ 0\end{array}\right) \delta^{f_{i}}(r)=\delta_{i}(r)$. Thus, $r \in \operatorname{ker}\left(\delta_{i}\right)$. Hence $r \in R_{\Delta}$.

(e) This follows immediately from $(d)$.

(f) If $R$ is $\mathbb{N}^{(I)}$-simple, then clearly $R$ is also $\Delta$-simple. Now suppose that $R$ is not $\mathbb{N}^{(I)}$ simple. We want to show that $R$ is not $\Delta$-simple. Let $J$ be a non-zero proper $\mathbb{N}^{(I)}$-invariant ideal of $R$. Take $i \in I$. Define $f_{i} \in \mathbb{N}^{(I)}$ as in the proof of (c). For any $r \in J$ we get $\delta_{i}(r)=\left(\begin{array}{c}f_{i} \\ 0\end{array}\right) \delta^{f_{i}}(r) \in J$. This shows that $J$ is $\Delta$-invariant and hence $R$ is not $\Delta$-simple.

Definition 22. Now we will define a well-order $\preceq$ on $\mathbb{N}^{(I)}$ which extends $\leq$. To this end, take $f, g \in \mathbb{N}^{(I)}$ with $f \neq g$ and put $|f|=\sum_{i \in I} f(i)$. Case 1: if $|f|>|g|$, then put $f \succ g$. Case 2: if $|f|<|g|$, then put $f \prec g$. Case 3: Suppose that $|f|=|g|$. Then there is $j \in I$ such that $f(i)=g(i)$, for $i \gg j$, but $f(j) \neq g(j)$. If $f(j)>g(j)$, then put $f \succ g$. If $f(j)<g(j)$, then put $f \prec g$. We will refer to $\preceq$ as the graded lexicographical ordering on $\mathbb{N}^{(I)}$

Remark 23. For each $i \in I$, the map $\delta_{i}: R \rightarrow R$ may be extended to an additive map $\tilde{\delta}_{i}: S \rightarrow S$ by defining $\tilde{\delta}_{i}\left(\sum_{f \in \mathbb{N}^{(I)}} r_{f} x^{f}\right)=\sum_{f \in \mathbb{N}^{(I)}} \delta_{i}\left(r_{f}\right) x^{f}$.

Theorem 24. If $\Delta$ is a commutative set of left (right) kernel derivations on $R$, then the differential monoid ring $S=R\left[\mathbb{N}^{(I)} ; \pi\right]$ is simple if and only if $R$ is $\Delta$-simple and $Z(S)$ is a field.

Proof. Put $G=\mathbb{N}^{(I)}$ and equip $G$ with the graded lexicographical ordering. All ideals $J$ of $S$ are $G$-invariant. Indeed, take $s=\sum_{f \in G} r_{f} x^{f} \in J$ and $i \in I$. Then $J \ni x^{f_{i}} s-s x^{f_{i}}=$ $\sum_{f \in G} \delta_{i}\left(r_{f}\right) x^{f}=\tilde{\delta}_{i}(s)$. By induction, $\pi_{g}^{f}(s) \in J$ for all $f, g \in G$ with $f \geq g$. This implies that $G$-simplicity of $S$ is equivalent to simplicity of $S$. Also, $Z(S)^{G}=Z(S)$. In fact, given $s=\sum_{f \in G} r_{f} x^{f} \in Z(S)$ and $i \in I$, we have $0=x^{f_{i}} s-s x^{f_{i}}=\sum_{f \in G} \delta_{i}\left(r_{f}\right) x^{f}=\tilde{\delta}_{i}(s)$. The claim now follows immediately from Theorem 18 and Proposition [21(f).

Remark 25. Theorem 24 generalizes [24, Theorem 4.15] both to the case of several variables and to the non-associative situation. In fact, if $R$ is associative, $I$ is the finite set $\{1, \ldots, n\}$ and each $\delta_{i}$, for $i \in I$, is a derivation on $R$, then $R\left[\mathbb{N}^{(I)} ; \pi\right]$ coincides with the differential polynomial ring $R\left[x_{1}, \ldots, x_{n} ; \delta_{1}, \ldots, \delta_{n}\right]$ (see Section 11). 
Now we wish to proceed to prove non-associative generalizations (see Theorem 33 and Theorem 35) of results by Voskoglou [28] and Malm 21] for differential polynomial rings in, possibly, infinitely many variables. Therefore, for the rest of this section, we assume that $\Delta$ is a commutative set of left (or right) kernel derivations on a (possibly non-associative) ring $R$ and we put $S=R\left[\mathbb{N}^{(I)} ; \pi\right]$. To this end, we first prove a few useful propositions.

Proposition 26. If $f, g, h \in \mathbb{N}^{(I)}$ are chosen so that $f=g+h$, then, for every $l \in \mathbb{N}^{(I)}$, we get that $\sum_{p+q=l}\left(\begin{array}{l}g \\ p\end{array}\right)\left(\begin{array}{l}h \\ q\end{array}\right)=\left(\begin{array}{l}f \\ l\end{array}\right)$.

Proof. This follows from Vandermonde's identity: $\sum_{q+r=s}\left(\begin{array}{l}b \\ q\end{array}\right)\left(\begin{array}{l}c \\ r\end{array}\right)=\left(\begin{array}{l}a \\ s\end{array}\right)$ which holds for all $a, b, c, q, r, s \in \mathbb{N}$.

Definition 27. If $f \in \mathbb{N}^{(I)}$, then we put $(-1)^{f}=(-1)^{|f|}$.

Proposition 28. If $r \in R$ and $f \in \mathbb{N}^{(I)}$, then $r x^{f}=\sum_{g \leq f}(-1)^{g}\left(\begin{array}{l}f \\ g\end{array}\right) x^{f-g} \delta^{g}(r)$.

Proof. Suppose that the cardinality of the support of $f$ is $n$. We will show the claim by induction over $n$.

Base case: $n=1$. This has already been proven in [23, Proposition 3.7].

Induction step: Suppose that $n>1$ and that the claim holds for all elements in $\mathbb{N}^{(I)}$ such that the cardinality of the support of the element is less than $n$. Suppose that $f=g+h$, for some $g, h \in \mathbb{N}^{(I)}$, is chosen so that the cardinalities of the supports of $g$ and $h$ are less than $n$. From the induction hypothesis and Proposition 26, we now get that

$$
\begin{aligned}
r x^{f} & =r x^{g+h}=\left(r x^{g}\right) x^{h}=\sum_{p \leq g}(-1)^{p}\left(\begin{array}{l}
g \\
p
\end{array}\right) x^{g-p} \delta^{p}(r) x^{h} \\
& =\sum_{p \leq g, q \leq h}(-1)^{p}(-1)^{q}\left(\begin{array}{l}
g \\
p
\end{array}\right)\left(\begin{array}{l}
h \\
q
\end{array}\right) x^{g-p} x^{h-q}\left(\delta^{p} \circ \delta^{q}\right)(r) \\
& =\sum_{p \leq g, q \leq h}(-1)^{p+q}\left(\begin{array}{l}
g \\
p
\end{array}\right)\left(\begin{array}{l}
h \\
q
\end{array}\right) x^{f-(p+q)} \delta^{p+q}(r) \\
& =\sum_{l \leq f} \sum_{p+q=l}(-1)^{l}\left(\begin{array}{l}
g \\
p
\end{array}\right)\left(\begin{array}{l}
h \\
q
\end{array}\right) x^{f-l} \delta^{l}(r)=\sum_{l \leq f}(-1)^{l}\left(\begin{array}{l}
f \\
l
\end{array}\right) x^{f-l} \delta^{l}(r) .
\end{aligned}
$$

Proposition 29. If $f, g, h \in \mathbb{N}^{(I)}$, then $\left(\begin{array}{c}f \\ g\end{array}\right)\left(\begin{array}{c}f-g \\ h\end{array}\right)=\left(\begin{array}{c}f \\ h\end{array}\right)\left(\begin{array}{c}f-h \\ g\end{array}\right)$.

Proof. This follows from the well known equality $\left(\begin{array}{l}a \\ b\end{array}\right)\left(\begin{array}{c}a-b \\ c\end{array}\right)=\left(\begin{array}{c}a \\ c\end{array}\right)\left(\begin{array}{c}a-c \\ b\end{array}\right)$ which holds for all $a, b, c \in \mathbb{N}$.

Proposition 30. If $a \in Z(S)$, then, for each $g \in \mathbb{N}^{(I)}$, we get that $\sum_{f \geq g} x^{f-g}\left(\begin{array}{l}f \\ g\end{array}\right) a_{f} \in Z(S)$.

Proof. Put $b=\sum_{f \geq g} x^{f-g}\left(\begin{array}{l}f \\ g\end{array}\right) a_{f}$. Using Proposition 21(e), it is enough to check the conditions in Corollary 10. Since $a$ commutes with every $x^{h}$, for $h \in \mathbb{N}^{(I)}$, we get that $a_{f} \in R_{\Delta}$, for $f \in \mathbb{N}^{(I)}$. Next we show that $b$ commutes with every $r \in R$. Using that $a_{f} \in R_{\Delta}$, for 
$f \in \mathbb{N}^{(I)}$, we can write $a=\sum_{f \in \mathbb{N}^{(I)}} x^{f} a_{f}$. Since $a r=r a$, we may use Proposition 28 to conclude that

$$
\begin{aligned}
\sum_{f} x^{f} a_{f} r & =a r=r a=\sum_{f} r x^{f} a_{f}=\sum_{f, g}(-1)^{g}\left(\begin{array}{l}
f \\
g
\end{array}\right) x^{f-g} \delta^{g}(r) a_{f} \\
& =\sum_{f, h}(-1)^{f-h}\left(\begin{array}{c}
f \\
f-h
\end{array}\right) x^{h} \delta^{f-h}(r) a_{f},
\end{aligned}
$$

where we in the last sum have put $f-g=h$. Hence, for each $h \in \mathbb{N}^{(I)}$, we get that

$$
a_{h} r=\sum_{f}(-1)^{f-h}\left(\begin{array}{c}
f \\
f-h
\end{array}\right) \delta^{f-h}(r) a_{f}
$$

Thus $r b=\sum_{f} r x^{f-g}\left(\begin{array}{l}f \\ g\end{array}\right) a_{f}=\sum_{f, h} x^{f-g-h}(-1)^{h}\left(\begin{array}{c}f-g \\ h\end{array}\right) \delta^{h}(r)\left(\begin{array}{l}f \\ g\end{array}\right) a_{f}$. If we now put $v=f-h$ in the last sum and use Proposition 29, then we get

$$
\sum_{f, v} x^{v-g}\left(\begin{array}{l}
f \\
g
\end{array}\right)\left(\begin{array}{l}
f-g \\
f-v
\end{array}\right)(-1)^{f-v} \delta^{f-v}(r) a_{f}=\sum_{f, v} x^{v-g}\left(\begin{array}{c}
f \\
f-v
\end{array}\right)\left(\begin{array}{l}
v \\
g
\end{array}\right)(-1)^{f-v} \delta^{f-v}(r) a_{f} .
$$

Finally, using (3), the last sum equals $\sum_{v} x^{v-g}\left(\begin{array}{l}v \\ g\end{array}\right) a_{v} r=b r$.

To conclude the proof, we show that $b$ associates with all elements in $R$. From the relations $(R, R, a)=\{0\}$ and $(a, R, R)=\{0\}$ it follows that for each $f \in \mathbb{N}^{(I)},\left(R, R, a_{f}\right)=$ $\left(a_{f}, R, R\right)=\{0\}$. Hence we get that $\left(R, R, b_{f}\right)=\left(b_{f}, R, R\right)=\{0\}$, for $f \in \mathbb{N}^{(I)}$. Thus, $(b, R, R)=(R, R, b)=\{0\}$. Since $[b, R]=\{0\}$, we automatically get that $(R, b, R)=\{0\}$ (see the proof of [23, Proposition 2.1]).

Definition 31. Take $a=\sum_{f \in \mathbb{N}^{(I)}} a_{f} x^{f} \in S$. Recall from Definition 17 that $\operatorname{deg}(a)$ is the largest $f \in \mathbb{N}^{(I)}$, with respect to $\preceq$, such that $a_{f} \neq 0$. If $a$ is non-zero and $a_{\operatorname{deg}(a)}=1$, then we say that $a$ is monic. We say that $a$ is constant if $\operatorname{deg}(a)=0$. We say that $a$ is linear if $a$ is non-constant, $a_{0}=0$ and the set $\operatorname{supp}(\operatorname{deg}(a))$ contains exactly one element. Notice that if $f \in \mathbb{N}^{(I)}$ has $\operatorname{supp}(f)=\{i\}$ for some $i \in I$, then, for every $r \in R$, the relation $x^{f} r=\delta_{i}(r)+r x^{f}$ holds.

Proposition 32. Suppose that $R$ is $\Delta$-simple and that $\operatorname{char}(R)=0$. Put $F=Z(R)_{\Delta}$. The following assertions hold:

(a) If $Z(S)$ only contains constants, then $Z(S)=F$.

(b) If $Z(S)$ contains non-constants, then $Z(S)$ contains a unique, up to addition by elements from $F$, non-constant monic a of least graded lexicographical degree. In that case, $Z(S)$ is not a field and there is $c \in R_{\Delta}, m \in \mathbb{N}$ and $c_{i} \in F$, for $i \in$ $\{1, \ldots, m-1\}$, such that $a=x_{m}+\sum_{i=1}^{m-1} c_{i} x_{i}-c$ and, hence, $\delta_{c}=\delta_{m}+\sum_{i=0}^{m-1} c_{i} \delta_{i}$.

Proof. (a) This is clear.

(b) Suppose that $Z(S)$ is not contained in $R$. Let $a$ be non-constant in $Z(S)$ of least graded lexicographical degree $h \in \mathbb{N}^{(I)}$. For each $g \in \mathbb{N}^{(I)}$, let $b_{g}=\sum_{f \geq g} x^{f-g}\left(\begin{array}{l}f \\ g\end{array}\right) a_{f}$. From Proposition 30 and the definition of $a$ it follows that if $g>0$, then $a_{g}=b_{g} \in Z(S)$. Thus, 
if $g>0$, then $a_{g} \in F$. In particular, $a_{h} \in F \backslash\{0\}$. We can thus, from now on, assume that $a_{h}=1$ so that $a$ is monic. We claim that $a-a_{0}$ is linear. If we assume that the claim holds, then, by the definition of the graded lexicographical ordering, there is $c \in R_{\Delta}$, $m \in \mathbb{N}$ and $c_{i} \in F$, for $i \in\{1, \ldots, m-1\}$, such that $a=x_{m}+\sum_{i=1}^{m-1} c_{i} x_{i}-c$. From the relations $a r=r a$, for $r \in R$, it follows that $\delta_{c}=\delta_{m}+\sum_{i=0}^{m-1} c_{i} \delta_{i}$. Now we show the claim. Seeking a contradiction, suppose that $\operatorname{supp}(\operatorname{deg}(a))$ contains more than one element. Then there is $g \in \mathbb{N}^{(I)}$ such that $h>g>0$. Since $\operatorname{char}(R)=0$, we get that $\left(\begin{array}{l}h \\ g\end{array}\right) \in F \backslash\{0\}$ and thus $0<\operatorname{deg}\left(b_{g}\right)<\operatorname{deg}(a)$ which contradicts the choice of $a$.

Theorem 33. If $\operatorname{char}(R)=0$ and we put $F=Z(R)_{\Delta}$, then $S$ is simple if and only if $R$ is $\Delta$-simple and no non-trivial finite $F$-linear combination of elements from $\Delta$ is an inner derivation on $R$ defined by an element from $R_{\Delta}$.

Proof. The "if" statement follows from Theorem 24 and Proposition 32. Now we show the "only if" statement. Suppose that $S$ is simple. By Theorem 24, $R$ is $\Delta$-simple and $Z(S)$ is a field. Suppose that there are $c_{1}, \ldots, c_{n} \in F$, not all of them equal to zero, such that $c_{1} \delta_{1}+\ldots+c_{n} \delta_{n}=\delta_{c}$. Take $c \in R_{\Delta}$ and consider the element $p=c_{1} x_{1}+\ldots c_{n} x_{n}-c$. Then it is clear that $p x_{i}=x_{i} p$ for all $i \in I$. Now take $r \in R$. Then $p r=r p+r c+c_{1} \delta_{1}(r)+\ldots+$ $c_{n} \delta_{n}(r)-c r=r p+c_{1} \delta_{1}(r)+\ldots+c_{n} \delta_{n}(r)+r c-c r=r p+c_{1} \delta_{1}(r)+\ldots+c_{n} \delta_{n}(r)-\delta_{c}(r)=r p$. Therefore $p \in Z(S)$. But, by Proposition 32 , this is a contradiction since $Z(S)$ is a field and $p$ is non-constant.

In the proof of the next proposition, we will use the following notation. Let $N=$ $\{-\infty\} \cup \mathbb{N}$ and let $p$ be a prime number. We will formally write $p^{-\infty}=0$. Let $N^{(I)}$ denote the set of functions $f: I \rightarrow N$ with the property that $f(i)=-\infty$ for all but finitely many $i \in I$. Given $f \in N^{(I)}$, let $p^{f} \in \mathbb{N}^{(I)}$ be defined by $\left(p^{f}\right)(i)=p^{f(i)}$, for $i \in I$.

Proposition 34. Suppose that $R$ is $\Delta$-simple and that $\operatorname{char}(R)=p$. Put $F=Z(R)_{\Delta}$. The following assertions hold:

(a) If $Z(S)$ only contains constants, then $Z(S)=F$.

(b) If $Z(S)$ contains non-constants, then $Z(S)$ contains a unique, up to addition by elements from $F$, non-constant monic a of least graded lexicographical degree. In that case, if the maps $\delta_{i}^{p^{j}}$, for $i \in I$ and $j \in \mathbb{N}$, are $F$-linearly independent, then there is $c \in R_{\Delta}, m, n \in \mathbb{N}$ and $c_{i j} \in F$, for $(i, j) \in\{1, \ldots, m\} \times\{0, \ldots, n\}$, such that $c_{m n}=1$ and $a=\sum_{i=1}^{m} \sum_{j=0}^{n} c_{i j} x_{i}^{p^{j}}-c$. Thus, $\delta_{c}=\sum_{i=1}^{m} \sum_{j=0}^{n} c_{i j} \delta_{i}^{p^{j}}$.

Proof. (a) This is clear.

(b) Suppose that $Z(S)$ is not contained in $R$. Let a be a non-constant polynomial in $Z(S)$ of least graded lexicographical degree $h \in \mathbb{N}^{(I)}$. For each $g \in \mathbb{N}^{(I)}$, let $b_{g}=$ $\sum_{f \geq g} x^{f-g}\left(\begin{array}{l}f \\ g\end{array}\right) a_{f}$. From Proposition 30 and the definition of $a$ it follows that if $g>0$, then $a_{g}=b_{g} \in Z(S)$. Thus, if $g>0$, then $a_{g} \in F$. In particular, $a_{h} \in F \backslash\{0\}$. We can thus, from now on, assume that $a_{h}=1$ so that $a$ is monic. Take $f \in \mathbb{N}^{(I)}$ such that $a_{f} \neq 0$. From Proposition 30 and Lucas' theorem it follows that $f=p^{t}$ for some $t \in N^{(I)}$. We say that $t$ is more than singly supported if there are $i, j \in I$, with $i<j$, such 
that $t(i) \neq-\infty \neq t(j)$. Seeking a contradiction, suppose that the set $Z=\left\{t \in N^{(I)} \mid\right.$ $t$ is more than singly supported and $\left.a_{p^{t}} \neq 0\right\}$ is non-empty. To this end, let $s$ denote the unique element from $Z$ of least graded lexicographical degree. Thus, there are $i, j \in I$, with $i<j$, such that $s(i) \neq-\infty \neq s(j)$ and $s(k)=-\infty$, for $k>j$. Given $n \in N$, define $s_{n} \in N^{(I)}$ by the relations $s_{n}(l)=s(l)$, for $l<j$, and $s_{n}(j)=n$, otherwise. In the relation $a r-r a=0$, considering the coefficient of $x^{p^{s}-\infty}$ yields the relation $\sum_{n=0}^{j} a_{p^{s_{n}}} \delta_{n}^{p^{s_{n}(j)}}=0$ which is a contradiction since $a_{p^{s_{j}}} \neq 0$. Hence $Z=\emptyset$ and the desired result follows.

Theorem 35. If $\operatorname{char}(R)=p$ and we put $F=Z(R)_{\Delta}$, then $S$ is simple if and only if $R$ is $\Delta$-simple and no non-trivial finite $F$-linear combination of $\delta_{i}^{p^{j}}$, for $i \in I$ and $j \in \mathbb{N}$, is an inner derivation on $R$ defined by an element from $R_{\Delta}$.

Proof. The "if" statement follows from Theorem 24 and Proposition 34. Now we show the "only if" statement. Suppose that $S$ is simple. By Theorem 24, $R$ is $\Delta$-simple and $Z(S)$ is a field. Seeking a contradiction, suppose that there are $c_{i j} \in F$, not all of them equal to zero, and $c \in R_{0}$ such that $\sum_{i j} c_{i j} \delta_{i}^{p^{j}}=\delta_{c}$. Consider now the element $a=\sum_{i j} c_{i}^{p^{j}} x_{i}^{p^{j}}-c$. Then it is clear that $a x_{i}=x_{i} a$ for all $i \in I$. Take $r \in R$. Then $a r-r a=r c+c_{1} \delta_{1}(r)+$ $\ldots+c_{n} \delta_{n}(r)-c r=c_{1} \delta_{1}(r)+\ldots+c_{n} \delta_{n}(r)+r c-c r=c_{1} \delta_{1}(r)+\ldots+c_{n} \delta_{n}(r)-\delta_{c}(r)=0$. Therefore $a \in Z(S)$. But, by Proposition 34, this is a contradiction since $Z(S)$ is a field and $a$ is non-constant.

\section{REFERENCES}

[1] S. Amitsur, Finite differential polynomials, Riveon Lematematika 4, 1-8 (1950).

[2] S. A. Amitsur, Derivations in simple rings, Proc. London Math. Soc. (3) 7, 87-112 (1957).

[3] G. M. Bergman, A ring primitive on the right but not on the left, Proc. Amer. Math. Soc. 15, 473-475 (1964).

[4] G. Cauchon, Idéaux bilatères et centre des anneaux de polynômes de Ore sur les anneaux quasisimples, Séminaire d'Algèbre Paul Dubreil 31ème année (Paris, 1977-1978), pp. 397-407, Lecture Notes in Math., 740, Springer, Berlin (1979).

[5] P. M. Cohn, Quadratic extensions of skew fields, Proc. London Math. Soc. (3) 11, 531-556 (1961).

[6] P. M. Cohn, Skew field constructions, London Mathematical Society Lecture Notes Series, No. 27, Cambridge University Press, Cambridge-New York-Melbourne (1977).

[7] E. P. Cojuhari, Monoid algebras over non-commutative rings, Int. Electron. J. Algebra 2, 28-53 (2007).

[8] E. P. Cojuhari and B. J. Gardner, Generalized higher derivations, Bull. Aust. Math. Soc. 86(2), 266-281 (2012).

[9] E. P. Cojuhari and B. J. Gardner, Skew ring extensions and generalized monoid rings, Acta Math. Hungar. 154(2), 343-361 (2018).

[10] J. Cozzens and C. Faith, Simple Noetherian rings, Cambridge Tracts in Mathematics, No. 69, Cambridge University Press, Cambridge-New York-Melbourne (1975).

[11] K. R. Goodearl, Centralizers in differential, pseudodifferential, and fractional differential operator rings, Rocky Mountain J. Math. 13(4), 573-618 (1983).

[12] H. Hasse and F. K. Schmidt, Noch eine Begründung der Theorie der höheren Differentialquotienten in einem algebraischen Funktionenkörper einer Unbestimmten, J. Reine Angew. Math. 177, 215-237 (1937).

[13] L. Hellström and S. D. Silvestrov, Commuting elements in q-deformed Heisenberg algebras, World Scientific Publishing Co., River Edge, NJ (2000). 
[14] N. Jacobson, Pseudo-linear transformations, Ann. of Math. (2) 38(2), 484-507 (1937).

[15] N. Jacobson, Finite-dimensional division algebras over fields, Springer-Verlag, Berlin (1996).

[16] D. A. Jordan, Ore extensions and Jacobson rings, Ph.D. thesis, University of Leeds (1975).

[17] T. Y. Lam and A. Leroy, Principal one-sided ideals in Ore polynomial rings, Algebra and its applications (Athens, OH, 1999), 333-352, Contemp. Math. 259, Amer. Math. Soc., Providence, RI (2000).

[18] T. Y. Lam, A. Leroy and J. Matczuk, Primeness, semiprimeness and prime radical of Ore extensions, Comm. Algebra 25(8), 2459-2506 (1997).

[19] A. Leroy and J. Matczuk, Prime ideals of Ore extensions, Comm. Algebra 19(7), 1893-1907 (1991).

[20] A. Leroy and J. Matczuk, The extended centroid and $X$-inner automorphisms of Ore extensions, $J$. Algebra 145(1), 143-177 (1992).

[21] D. R. Malm, Simplicity of partial and Schmidt differential operator rings, Pacific J. Math. 132(1), 85-112 (1988).

[22] J. C. McConnell and J. C. Robson, Noncommutative Noetherian rings, Graduate Studies in Mathematics, Vol. 30, American Mathematical Society, Providence, RI (2001).

[23] P. Nystedt, J. Öinert and J. Richter, Non-associative Ore extensions, Israel J. Math. 224(1), 263-292 (2018).

[24] J. Öinert, J. Richter and S. D. Silvestrov, Maximal commutative subrings and simplicity of Ore extensions, J. Algebra Appl. 12(4), 1250192, 16 pp. (2013).

[25] O. Ore, Theory of non-commutative polynomials, Ann. of Math. (2) 34(3), 480-508 (1933).

[26] L. H. Rowen, Ring Theory, Vol. I, Pure and Applied Mathematics, No. 127, Academic Press, Boston, MA (1988).

[27] T. H. M. Smits, Skew Polynomial Rings, Indag. Math. 30, 209-224 (1968).

[28] M. G. Voskoglou, Simple skew polynomial rings, Publ. Inst. Math. (Beograd) (N.S.) 37(51), 37-41 (1985). 